\title{
Analysis of cracked plates using localized multi-domain differential quadrature method
}

\author{
Tharwat Osman ${ }^{1,}$, , Mohamed. S. Matbuly ${ }^{1}$, Salwa. A. Mohamed ${ }^{1}$, Mohamed Nassar ${ }^{2}$ \\ ${ }^{1}$ Dept. of Math. And Phys., Faculty of Engineering, Zagazig University, Zagazig, Egypt \\ ${ }^{2}$ Dept. of Math. And Phys., Faculty of Engineering, Cairo University, Giza, Egypt
}

Email address:

tharwatosman75@yahoo.com(T. Osman)

To cite this article:

Tharwat Osman, Mohamed. S. Matbuly, Salwa. A. Mohamed, Mohamed Nassar. Analysis of Cracked Plates Using Localized MultiDomain Differential Quadrature Method. Applied and Computational Mathematics. Vol. 2, No. 4, 2013, pp. 109-114. doi: $10.11648 /$ j.acm.20130204.12

\begin{abstract}
In this paper, A multi-domain differential quadrature method is employed to solve a mode III crack problem. The domain of the problem is assumed to be irregular rather than it possesses discontinuities, (cracks). The entire domain is divided into several subdomains, according to the crack locations. A conformal mapping is applied to transform the irregular subdomains to regular ones. Then the differential quadrature method is employed to solve the problem over the transformed domains. Further, it's focused on the crack regions by applying the localized version of differential quadrature method. The out of plane deflection is obtained at the immediate vicinity of the crack tips, such that the stress intensity factor can be calculated. The obtained results are compared with the previous analytical ones. Furthermore a parametric study is introduced to investigate the effects of elastic and geometric characteristics on the values of stress intensity factor.
\end{abstract}

Keywords: Irregular, Localized Differential Quadrature, Conformal Mapping, Mode III, Stress Intensity Factor

\section{Introduction}

Determinations of stress intensity factors, (SIFs), are one of the most important problems in fracture mechanics. This problem has been extensively studied by many authors using various analytical and numerical techniques. In general, there are two analytical techniques for solving such problems. The first one employees complex analysis while the second applies integral transformation with asymptotic analysis to reduce the problem to a system of singular integral equations [1-3]. The finite element method (FEM) and boundary element method (BEM) are the most frequently numerical techniques for solving crack problems. FEM needs to describe a special element at the immediate vicinity of the crack tips. The compatibility between this element and the others presents an additional problem. So, FEM is an expensive technique for solving crack problems [4-6]. Both the direct and indirect BEMs lead to a mathematical degeneracy when the crack tip is considered as a boundary node [7-9]. This difficulty has been overcome by several special BEMs. One of these methods based on dividing the cracked body into sub-regions along the cracks More recently such that each sub-domain does not contain any cracks. This method results in a larger matrix due to the extra nodes along the sub-region interfaces and may lead to ill-conditioned matrix.

More recently, the differential quadrature method (DQM), is introduced for solving several engineering problems, such that in thermodynamics, aerodynamics, structural and fracture mechanics. The method possesses the capability to achieve accurate results with a minimal computational effort [10-13], The classical version of DQM can't deal with discontinuous or irregular domains. So, a new version of DQM, (termed by multi-domain differential quadrature technique), is developed for solving discontinuity problems. The philosophy of multi-domain DQM inherits the merits of the flexibility of finite element method and at the same time retains the high accuracy of the DQM. The main advantages of multi-domain DQM can be summarized as follows: multi-domain DQM is able to deal with problems with either geometric or material discontinuity, therefore it is recommended for solving crack problems. Also it can deal with problems with doubly or multiply connected regions. Further, it is able to treat problems with inconsistent boundary conditions [14-18].

This work extends the applications of a multi-domain DQM to solve a mode III crack problem in an irregular plate. The strategy is to decompose the whole domain into 
several sub-domains according to crack branches. Then an appropriate conformal mapping is applied to transform each irregular sub-domain, (physical domain), to a regular one, (computational domain), such that DQM can be applied. The out of plane deflection is obtained at the immediate vicinity of the crack tips, such that the stress intensity factor can be determined. The obtained results well agreed with the available analytical ones. Further a parametric study is introduced to investigate the effects of elastic and geometric characteristics on the values of stress intensity factor.

\section{Formulation of the Problem}

Consider a cracked irregular plate made of isotropic material as in Fig. 1. The external boundaries of the plate are subjected to anti-plane shear tractions while the crack surfaces are free of tractions.

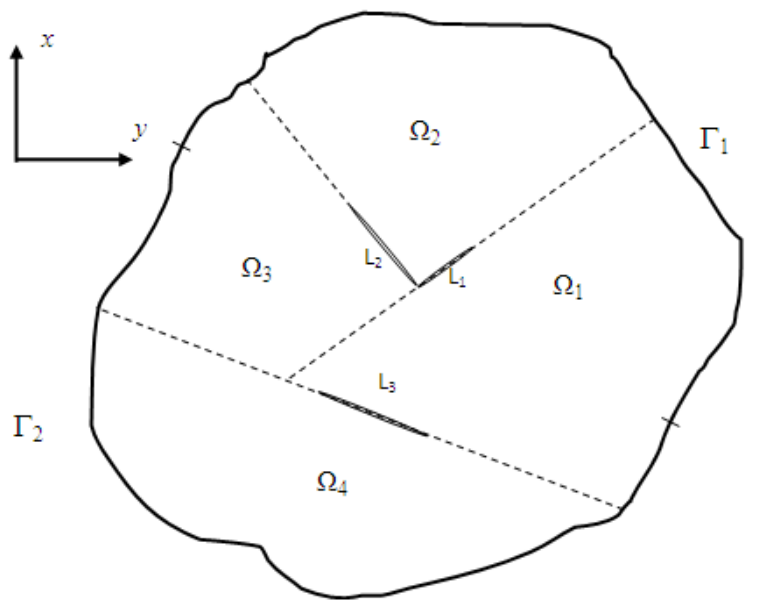

Fig. 1. Decomposition of the original domain

The equilibrium equation along the out of plane direction, $z$, can be written as:

$$
\tau_{i z, i}=0, \quad(i=x, y),
$$

Where, $\tau_{i z} ;(i=x \quad y)$ are the components of the antiplane shear stress.

The relation between the stress and out of plane displacement, $W$ is given by:

$$
\tau_{i z}=G W_{, i}, \quad(i=x, y)
$$

On substitution from Eq. 2 into Eq. 1 the equilibrium equation of the problem can be reduced to:

$$
W_{, i i}=0, \quad(i=x, y) .
$$

The external boundary conditions can be described as:

$$
\begin{aligned}
w(x, y)=\bar{w}(x, y), & (x, y) \in \Gamma_{1} \\
\tau_{x z}(x, y)=\bar{\tau}_{x}(x, y), & (x y) \in \Gamma_{2} \\
\tau_{y z}(x, y)=\bar{\tau}_{y}(x, y), & (x y) \in \Gamma_{2}
\end{aligned}
$$

where $\Gamma_{1} \cup \Gamma_{2}=\Gamma$ is the whole external boundaries of the plate. $W, \bar{\tau}_{x z}$ and $\bar{\tau}_{y z}$ are known functions.

Further the stresses along the crack surfaces can be described as:

$$
\lim _{(x, y) \rightarrow \pm L_{k}} \tau_{i z}(x, y)=0, \quad(i=x, y) \text { and }(k=1, n)
$$

where $L_{k}$ is location of $k^{\text {th }}$ branch of the crack. $n$ is the number of crack branches.

\section{Solution of the Problem}

The solution of the problem can be implemented through the following steps:

- Decompose the original domain, along the crack surfaces, into $m$ sub-domains as shown in Fig. 1.

- Employ appropriate geometric mapping to transform each irregular sub-domain in $x-y$ plane, (physical domain), to a rectangular one in $\xi-\eta$ plane, (computational domain), see Fig. 2.
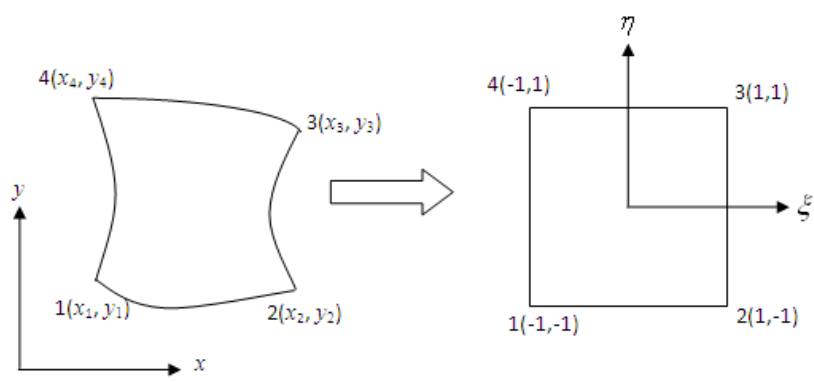

Fig. 2. Geometric transformation from the physical domain to a computational one.

This mapping may be done according to [19]:

Where $G$ is shear modulus.

$$
\begin{aligned}
{\left[\begin{array}{l}
x(\xi, \eta) \\
y(\xi, \eta)
\end{array}\right] } & =\left(\frac{1-\xi}{2}\right) S(-1, \eta)+\left(\frac{1+\xi}{2}\right) S(1, \eta)+\left(\frac{1-\eta}{2}\right) S(\xi,-1) \\
& +\left(\frac{1+\eta}{2}\right) S(\xi, 1)-\frac{(1-\xi)(1-\eta)}{4} S(-1,-1)-\frac{(1-\xi)(1+\eta)}{4} S(-1,1) \\
& -\frac{(1+\xi)(1-\eta)}{4} S(1,-1)-\frac{(1+\xi)(1+\eta)}{4} S(1,1)
\end{aligned}
$$


where the functions $S\left(\xi_{i}, \eta\right)$ and $S\left(\xi, \eta_{j}\right)$ represents the four boundary parametric curves of the original physical domain and $S\left(\xi_{i}, \eta_{j}\right)$ donates the $x$ and $y$ coordinates of the point corresponding to the coordinates $\left(\xi_{i}, \eta_{j}\right)$ in the computational space.

- $\quad$ For each sub-region, apply chain rule to transform the governing equations form $x-y$ system to that in $\xi-\eta$ one.

- Apply the method of differential quadrature to reduce the transformed governing equations to the following system of linear algebraic equations [2021]

$$
\sum_{k=1}^{N} C_{i, k}^{l} w_{k, j}^{l}+P_{l}^{2} \sum_{k=1}^{M} \bar{C}_{j, k}^{l} w_{i, k}^{l}=0 ; \quad l=1, m
$$

Where the super script $l$ refers to $l^{\text {th }}$ sub-domain, $(l=1, \mathrm{~m})$.

$P_{l}$ is a parameter resulted from geometric mapping of $l^{\text {th }}$ region from irregular shape to a regular one.

$C_{i, k}^{l}, \bar{C}_{j, k}^{l}$ are the weighting coefficient of the $2^{\text {nd }}$ order derivatives in $\xi$ and $\eta$-directions, respectively. $w_{i, k}^{l}$ are the unknown functional nodal values for each transformed subregion in $\xi-\eta$ plane.

The quadrature grid is designed with nodes as the roots of Chebyshev polynomials [20], such as:

$$
\begin{array}{ll}
\xi_{i}=-\cos \left(\frac{i-1}{N-1}\right) \pi, & i=1, N \\
\eta_{j}=-\cos \left(\frac{j-1}{M-1}\right) \pi, & j=1, M
\end{array}
$$

Where $N, M$ is the number of grid points in $\xi$ and $\eta$ directions, respectively

Along the interface of each adjacent sub-region, the continuity of conjunctiva nodes is also considered.

\section{Numerical Results}

A quadrature scheme is designed for solving mode III crack problems. The scheme suggests to initially solve the problem, over the entire domain by using a coarse grid then to refine the results, at immediate vicinity of the crack tips, over another, localized, fine grid as shown in Fig. 3, [22, 23].

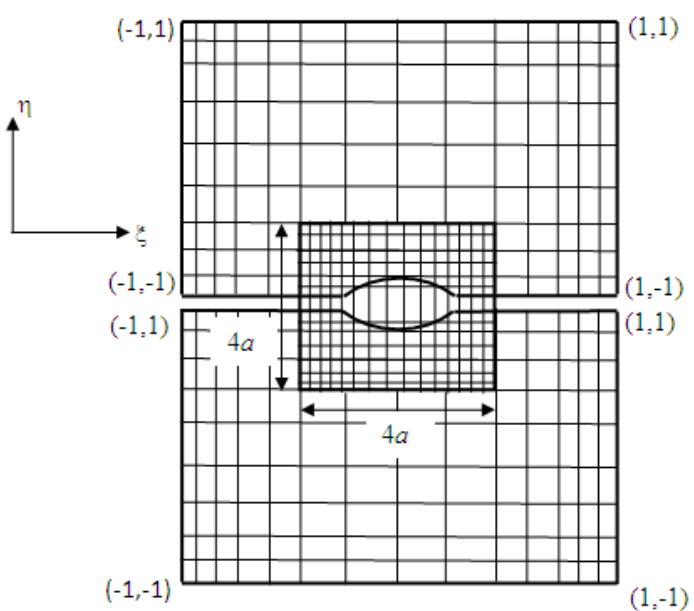

Fig. 3. Localized differential quadrature grid at the immediate vicinity of crack tips

Knowing the values of the anti-plane displacement, $W$, at immediate vicinity of the crack tips, the values of mode III stress intensity factors can be calculated as [24]:

$$
K_{I I I}=\frac{W(x, y) G}{\sin (\theta / 2) \sqrt{2 r / \pi}}
$$

where $\mathrm{G}$ is the shear modulus of the plate, $r$ is the distance from the crack tip to the position at which $K_{I I I}$ is

\begin{tabular}{|c|c|c|c|c|c|c|c|}
\hline $\begin{array}{l}\text { Crack } \\
\text { length } \\
\text { ratio: } \\
2 \text { a/D }\end{array}$ & Exact $K_{I I I}[24]$ & Obtained $\mathbf{K}_{\mathrm{III}}$ & $\begin{array}{l}\text { Error }= \\
\mid \frac{\text { obtaied } \mathrm{K}_{\mathrm{II}}-\text { exact } \mathrm{K}_{\mathrm{II}}}{\operatorname{exact}_{\mathrm{III}}}\end{array}$ & $\begin{array}{l}\text { Quadrature scher } \\
\text { Coarse grid } \\
\text { discretization : } \\
\mathrm{N}^{*} \mathrm{M}\end{array}$ & $\begin{array}{l}\text { Dimension of } \\
\text { localized grid }\end{array}$ & $\begin{array}{l}\text { Localized (fine) } \\
\text { grid } \\
\text { discretization : } \\
\mathrm{N}_{1} * \mathrm{M}_{1}\end{array}$ & $r$ \\
\hline 0.05 & 0.396741 & 0.395813 & 0.002339 & $21 * 21$ & $0.15 * 0.11$ & $15 * 13$ & 0.0087 \\
\hline 0.10 & 0.562822 & 0.568665 & 0.010381 & $21 * 21$ & $0.31 * 0.30$ & $18 * 13$ & 0.01 \\
\hline 0.15 & 0.692934 & 0.690811 & 0.003064 & $21 * 21$ & $0.31 * 0.40$ & $21 * 13$ & 0.0081 \\
\hline 0.20 & 0.806126 & 0.804046 & 0.002580 & $22 * 22$ & $0.50 * 0.50$ & $22 * 13$ & 0.013 \\
\hline 0.25 & 0.910180 & 0.904929 & 0.005769 & $25 * 25$ & $0.55 * 0.61$ & $26 * 13$ & 0.0128 \\
\hline 0.30 & 1.009481 & 1.012416 & 0.002907 & $27 * 27$ & $0.66 * 0.65$ & $26 * 13$ & 0.0162 \\
\hline 0.35 & 1.107069 & 1.091346 & 0.014202 & $28 * 28$ & $0.76 * 0.71$ & $29 * 13$ & 0.0174 \\
\hline
\end{tabular}
calculated. $\theta$ is the angle between the position and the direction of the crack, as shown in Fig.4.

Table 1. A comparative study: Variation of $K_{\text {III }}$ with crack length. 
For practical importance, the obtained results are normalized, (divided by $\tau_{0} \sqrt{D}$ ). Also, to ensure the validity of proposed scheme, the obtained results are compared with the previous analytical ones [24] as shown in table 1 .

$$
\text { Normalized } K_{I I I}(\text { exact })=\sqrt{2 \tan \left(\frac{a \pi}{D}\right)}
$$

Where D defines the width of the plate, as illustrated in Fig.4.

Further, a parametric study is introduced to investigate the effects of elastic and geometric characteristics of the problem on the values of normalized stress intensity factors.

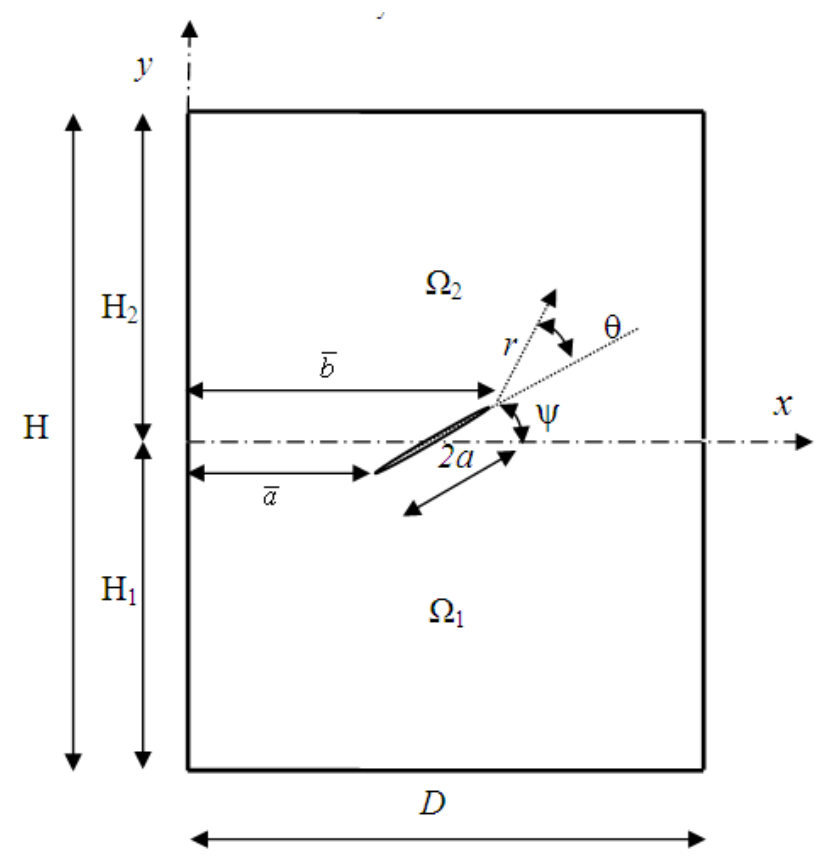

Fig. 4. Rectangular plate with crack.

Figures 5-7, 9 and 10 show that the values of $\mathrm{K}_{\mathrm{III}}$ increase with increasing of crack length. While, the values of $\mathrm{K}_{\mathrm{III}}$ decrease with increasing of aspect ratio: $\mathrm{H} / \mathrm{D}$, as shown in Fig. 5.

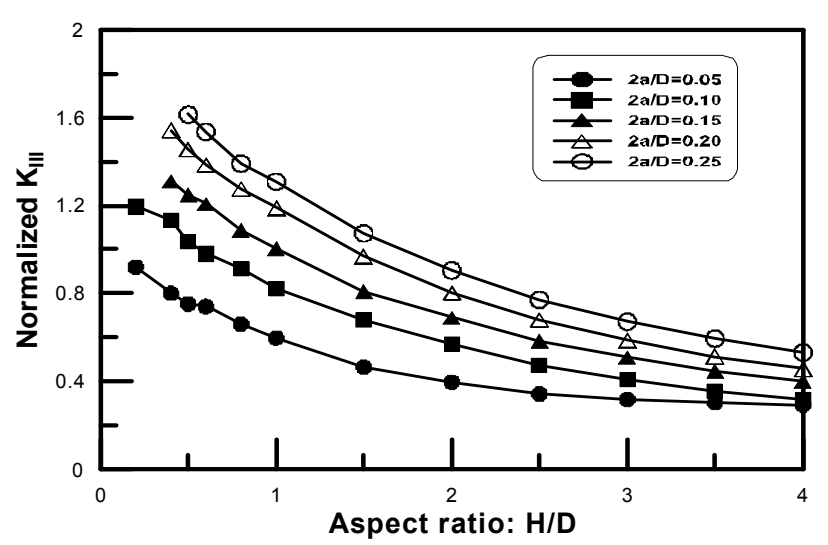

Fig. 5. Variation of normalized $K_{\text {III }}$ with the crack length and aspect ratio.
Also, Figure 6, shows that the values of $\mathrm{K}_{\mathrm{III}}$ decrease with increasing the distance between the crack surfaces and the boundaries.

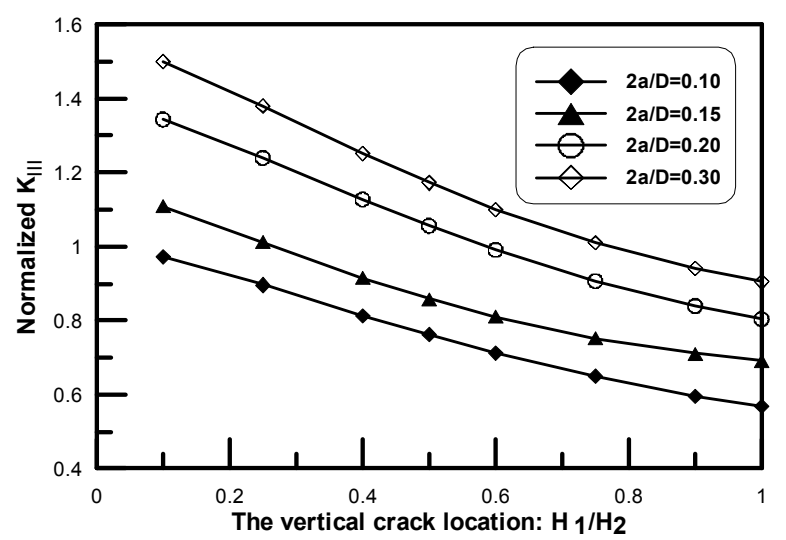

Fig. 6. Variation of normalized $K_{I I I}$ with the crack length and the vertical crack location.

The values of normalized $\mathrm{K}_{\mathrm{III}}$ slightly increases with increasing of crack orientation angle $\psi$ up to $\psi \approx 25^{\circ}$ then they decease beyond this value, as shown in Fig. 11. Also, the same behavior of the normalized $\mathrm{K}_{\mathrm{III}}$ is clearly reported for different radii of curvatures, as shown in Fig. 12.

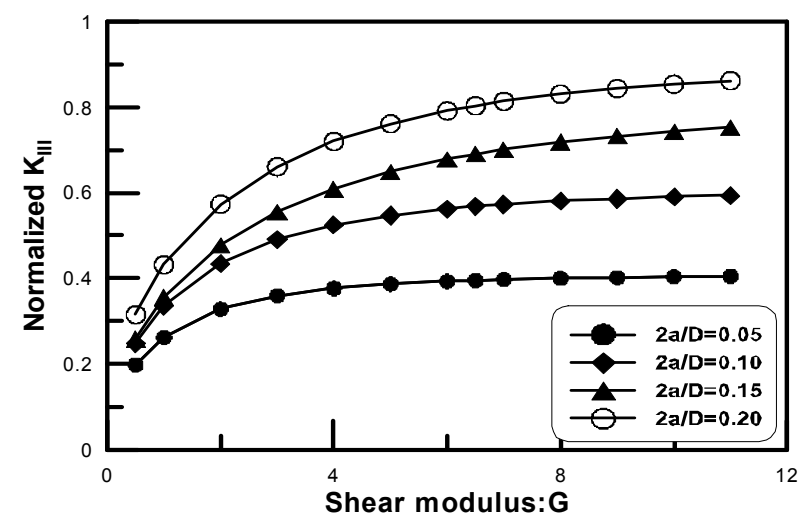

Fig. 7. Variation of normalized $K_{I I I}$ with the crack length and shear modulus.

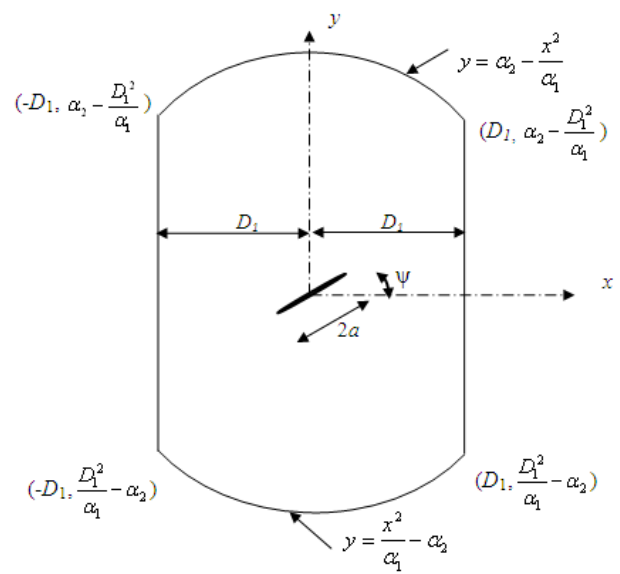

Fig. 8. Irregular plate with a crack 


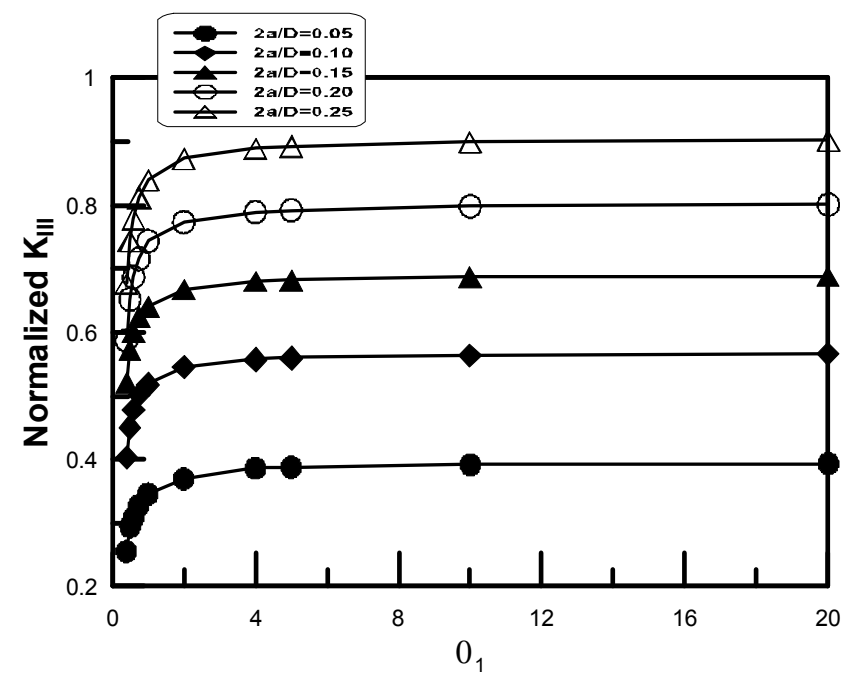

Fig. 9. Variation of normalized $K_{I I I}$ with the crack length and the radius of curvature.

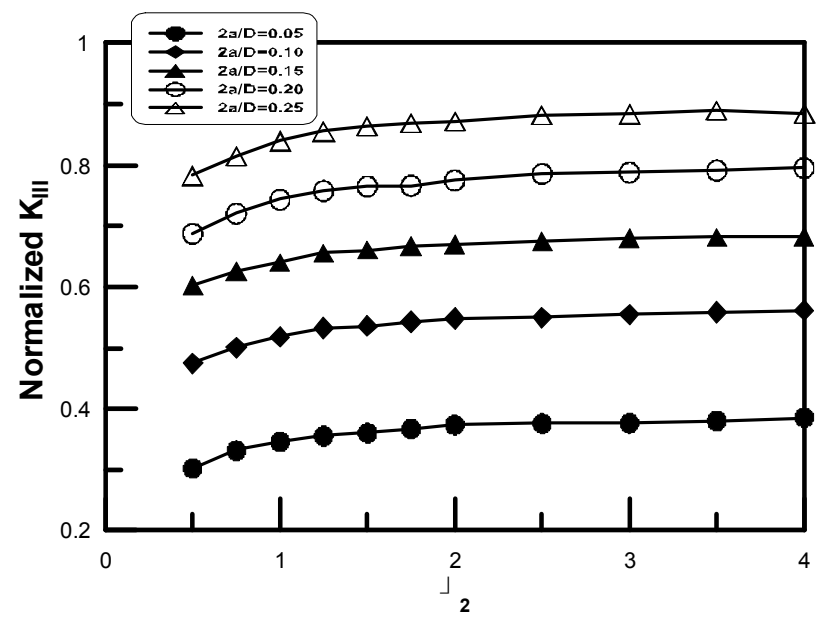

Fig. 10. Variation of normalized $K_{I I I}$ with the crack length and distance from the crack surfaces to the boundary apexes.

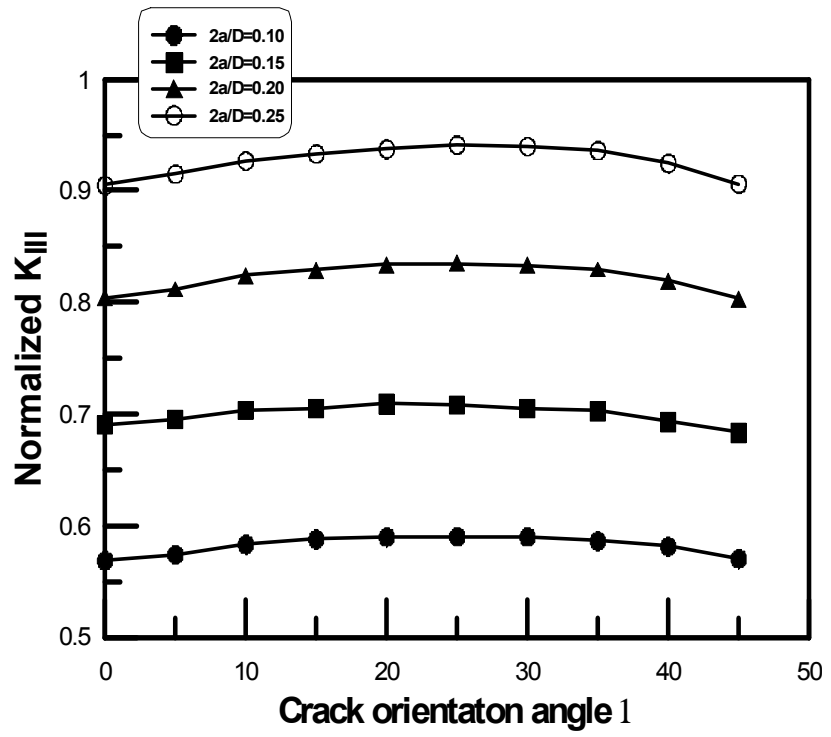

Fig. 11. Variation of normalized $K_{I I I}$ with crack orientation angle and crack length.

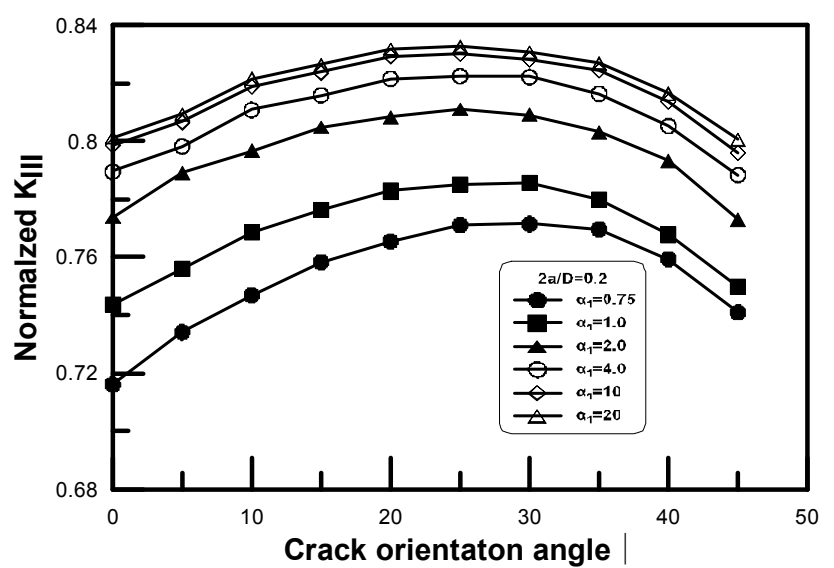

Fig. 12. Variation of normalized $K_{I I I}$ with crack orientation angle $\psi$ and the radius of curvature $\alpha_{1}$ at $2 a / D=0.2$.

\section{Conclusion}

A hybrid technique consists of multi-domain DQM and conformal mapping is applied for solving cracked irregular plate subjected to anti-plane shear loading. This work can be considered as an extension for the applications of DQM in discontinuity problems. The accuracy of the obtained results is achieved by comparing them with the previous ones. Further a parametric study is introduced to explain the influence of elastic and geometric characteristics on the values of stress intensity factor.

\section{References}

[1] Bora Yildirim, Özge Kutlu and Suat Kadioğlu, "Periodic crack problem for a functionally graded half-plane an analytic solution", International Journal of Solids and Structures, Vol. 48, pp. 3020-3031, 2011.

[2] H. G. Beom, J. W. Lee and C. B. Cui, ” Analysis of a kinked crack in an anisotropic material under antiplane deformation", Journal of Mechanical Science and Technology, Vol. 26, pp. 411-419, 2012.

[3] Jeong Woo Shin and Young-Shin Lee, “Anti-plane moving crack in a functionally graded piezoelectric layer between two dissimilar piezoelectric strips", Journal of Mechanical Science and Technology, Vol. 26, pp. 1017-1025, 2012.

[4] E. Wyart, D. Coulon, T. pardoen, J.F. Remacle and F. Lani, "Application of the sub- structured finite element/extended finite element method (S-FE/XFE) to the analysis of cracks in aircraft thin walled structures", Engineering Fracture Mechanics, Vol. 76, pp. 44-58, 2009.

[5] Jin-Shi Wen, Woo-Eon Ju, Tae-Kyung Han, Seung Tae Choil and Kyung-Sick Lee, "Finite element analysis of a subsurface penny-shaped crack with crack-face contact and friction under a moving compressive load", Journal of Mechanical Science and Technology, Vol. 26, pp. 27192726, 2012.

[6] Jingbo Duan, Yongjun Lei and Daokui Li, "Enriched finite element method for 2-D and 3-D blunt crack problems in a viscoelastic medium", Journal of Mechanical Science and Technology, Vol. 26, pp. 869-882, 2012. 
[7] Jun Lei, Felipe Garcia-Sanchez, Michael Wunsche, Chüanzeng, Yue-Sheng Wang and Andres Saez, " Dynamic analysis of interfacial crack problems in anisotropic bimaterials by a time-domain BEM", Engineering Fracture Mechanics, Vol. 76, pp. 1996-2010, 2009.

[8] X. Yan, B. Liu and J. Yu, "Cracks emanating from a square hole in rectangular plate in tension", Fatigue Fract Engng Mater Struct, Vol. 35, pp. 238-246, 2011.

[9] X. Yan and B. Liu, "Rectangular Tensile Sheet with Double Edge Notch Cracks", Fatigue Fract Engng Mater Struct, Vol. 35, pp. 466-475, 2012.

[10] Zhangxian Yuan and Xinwei Wang, "Buckling and postbuckling analysis of extensible beam-columns by using the differential quadrature method", Computers and Mathematics with Applications, Vol. 62, pp. 4499-4513, 2011.

[11] S. Hamed Meraji, Abbas Ghaheri and Parviz Malekzadeh, "An efficient algorithm based on the differential quadrature method for solving Navier-Stokes equations", International Journal for Numerical Methods in Fluids, (2012), Published online in Wiley Online Library (wileyonlinelibrary.com).

[12] Mohamed Nassar, Mohamed S. Matbuly and Ola Ragb,"Vibration analysis of structural elements using differential quadrature method", Journal of Advanced Research, Vol. 4, pp. 93-102, 2013.

[13] M. Mallakzadeh, A. A. Pasha Zanoosi and A. Alibeigloo, "Fundamental frequency analysis of microtubules under different boundary conditions using differential quadrature method", Communications in Nonlinear Science and Numerical Simulation, Vol. 18, No. 8, pp. 2240-2251, 2013.

[14] F. L. Liu and K.M. Liew, "Differential quadrature element method: a new approach for free vibration analysis of polar Mindlin plates having discontinuities", Comput. Methods Appl. Mech. Engrg., Vol. 179, pp. 407-423, 1999.

[15] F.-L. Liu, "Differential quadrature element method for buckling analysis of rectangular Mindlin plates having discontinuities", International Journal of Solids and Structures, Vol. 38, pp. 2305-2321, 2001.

[16] Hongzhi Zhong and Yuhong "A note on incorporation of domain decomposition into the differential quadrature method", Communications in Numerical Methods in Engineering Commun. Numer. Meth. Engng., Vol. 19, pp. 297-306, 2003.

[17] Xionghua Wu and YE Shen," Differential Quadrature Domain Decomposition Method for A Class Of Parabolic Equations", Computers And Mathematics With Applications, Vol. 48, pp. 1819-1832, 2004.

[18] Z. Zong, K. Y. Lam and Y. Y. Zhang, "A Multidomain Differential Quadrature Approach To Plane Elastic Problems With Material Discontinuty", Mathematical and Computer Modelling, Vol. 41, pp. 539-553, 2005.

[19] C. Shu,W. X. Wu, H. Ding and C. M. Wang, "Free Vibration Analysis of Curvilinear Quadrilateral Plates by the Differential Quadrature Method", Journal of Computational Physics, Vol. 163, pp. 452-466, 2000.

[20] Chang Shu,"Differential Quadrature and Its Application in Engineering", London springer, Verlag, 2000

[21] Liangliang Du and Xionghua Wu;" On a rational differential quadrature method in irregular domains for problems with boundary layers", Applied Mathematics and Computation, Vol. 218,pp. 1379-1388, 2011.

[22] C.H. Tsai, D. I. Young and C. C. Hsiang," The localized differential quadrature method for two-dimensional stream function formulation of Navier-Stokes equations ", Engineering Analysis with Boundary Elements, Vol. 35, pp. 1190-1203, 2011.

[23] M.E. Hamidi, M. R. Hashemi, N. Talebbeydokhti and S. P. Neill," numerical modeling of the mild slope equation using localized differential quadrature method", Ocean Engineering, Vol. 47, pp. 88-103, 2012.

[24] David J. Unger "Analytical fracture mechanics" Academic press, London, 1995. 